\title{
COSY - A COOLER SYNCHROTRON AND STORAGE RING
}

S.A. Martin, G.P.A. Berg, U. Hacker, A. Hardt, M. Köhler, J. Meißburger, F. Osterfeld, D. Prasuhn, G. Riepe, M. Rogge, O.W.B. Schult, J. Speth, P. Turek (Kernforschungsanlage Jiilich), G. Gaul (Univ. Muinster),

H. Hagedoorn, U.A. Van der Heide (Techn. Univ. Eindhoven), F. Hinterberger, M. Huber, R. Jahn, T. MayerKuckuk, (Univ. Bonn), H. Poth (CERN, Genf and KfK, Karlsruhe), H. Paetz gen. Schieck (Univ. Köln)

The storage ring cosy with phase space cooling and RF acceleration is designed to accept protons and light ions injected from the existing cyclotron JULIC or protons from the LINAC of the proposed neutron spallation source (SNQ). The lay-out of COSY was developed in cooperation with the Universities in Nordrhein-Westfalen and meets the experimental requirements of variable and high quality beams which are necessary for future nuclear research under discussion.

The motivation for the cooler-synchrotron COSy emerged from discussions of nuclear physicists in Nordrhein-Westfalen on future research activities. The "workshop on Electron Couling" in 1982 came to the conclusion that a cooler-synchrotron is an excellent tool for advanced nuclear research experiments. It should be built at the KFA in cooperation with the surrounding Universities. The three essential properties of the storage ring will be:

- high luminosities and very efficient use of the beam in the storage ring by thin internal targets

- energy variability in the range of $20 \mathrm{MeV}$ to $1.5 \mathrm{GeV}$ by RF acceleration

- very high beam quality through phase space cooling.

Two classes of experiments will be feasible in the first step of construction. At proton energies up to about $200 \mathrm{MeV}$ it will be possible to excite selectively fundamental nuclear spin isospin modes and to resolve the corresponding nuclear levels with the existing high resolution spectrometer BIG KARL ${ }^{2}$. At higher energies single nucleons in the nucleus can be excited internally and coherent meson production can be studied, processes which reflect details of the subnuclear structure.

\section{The Lattice}

The basic structure of COSY is a hexagon as shown in fig. 1. It consists of 6 unit cells which are connected by two long insertions built as telescopes to provide beam characteristics appropriate for the clectron cooler on one side and the high resolution target spot for the BIG KARL spectrometer on the other side. Each unit cell contains four $15^{\circ}$ bending magnets (B), two quadrupoles (F) and drift sections (O) in the following arrangement OFOBBOBBOFO.

Table 1 summarizes the main parameters of the lattice. The characteristic lattice functions $\left(B_{x}, \beta_{z}, 0\right)$ for the working point with a tune of $Q_{x}=$ 3.75 and $Q_{z}=3.30$ are displayed in fig. 2. For this working point and a dispersion of $\mathrm{D}_{\mathrm{T}}=-10 \mathrm{~m}$ at the target the transition is $\gamma_{t r}=1.0026$. Fig. 3 shows the dispersions along the ring for different working points and the corresponding $Y_{t r}$.

The target telescope provides variable dispersion $D_{T}$ at the target position (see fig. 4). This telescope allows four different target dispersion without changing the working point of the ring because

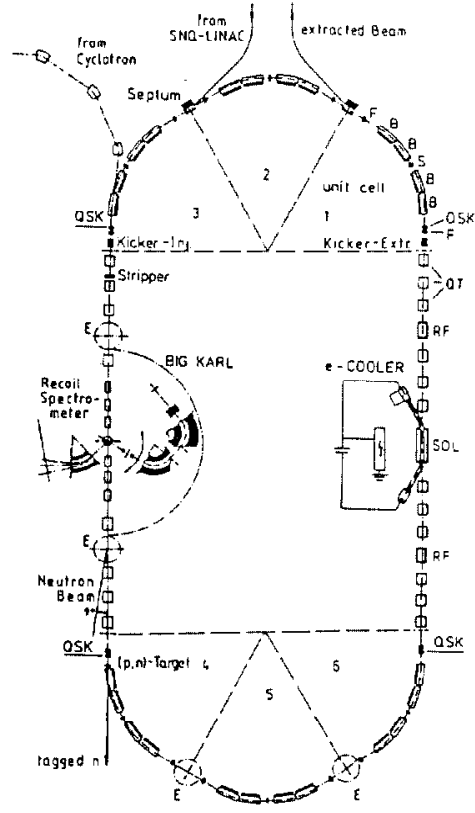

the tune shift of the insertions is always $2 \pi$ in both planes. This allows "dispersion mat ching" between ring and spectrometer for high resolution experiments. At the same time the Twiss parameter a can be varied in the range $-1.25 \leqslant \alpha<0.5$ so that "kinematic matching"2 between the ring and the spectrometer is possible up to $K$-values of $\lesssim 0.5$. This correction can be done with the telescope without changing the tune.

Figure 1:

cosy layout with 6 unit cells and two insertions $(35 \mathrm{~m})$.

\section{Table I: Parameters of the preliminary cosy lattice}

6 unit cells of the structure: OFOBBOBBOFO

Two tel escopes of 35 in length with magnification +

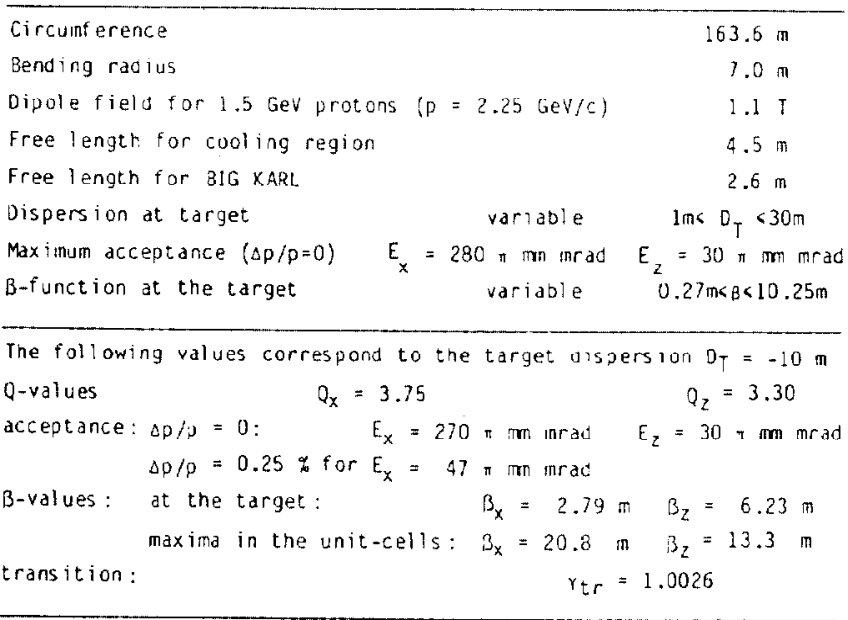

The polarization of particles with vertically transvers polarizations can be preserved by using a solenoid to counteract the spin precession in the cooler solenoid (SOL).

The purpose of the skewed quadrupoles (QSK) is to correct deviations of the dispersive plane from the horizontal plane. The sextupoles (S) between the dipole pairs allow chromaticity corrections. Octupoles take care for the generation of a tune spread if Landau damping ${ }^{3}$ is required. 


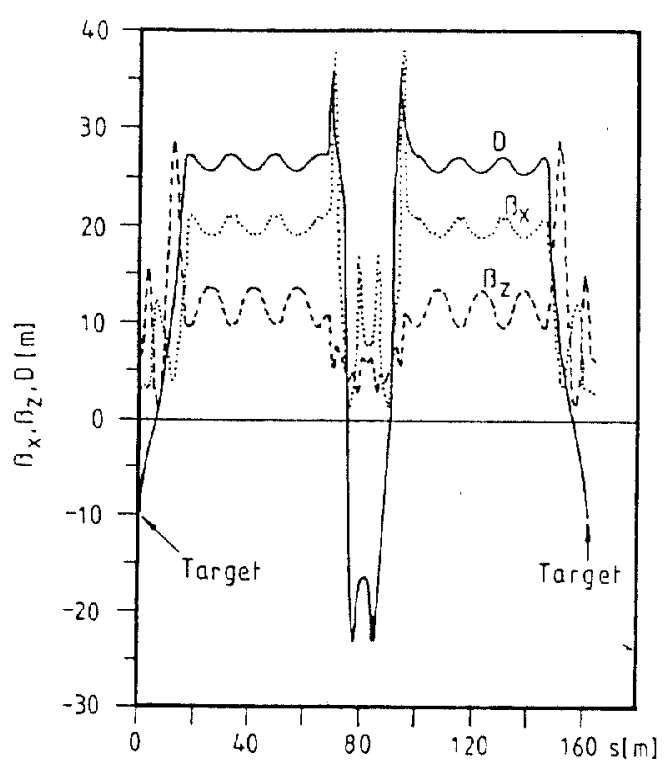

Figure 2: Lattice functions of CUSY for a working point with the dispersion $D_{T}=-10 \mathrm{~m}$ at the target.

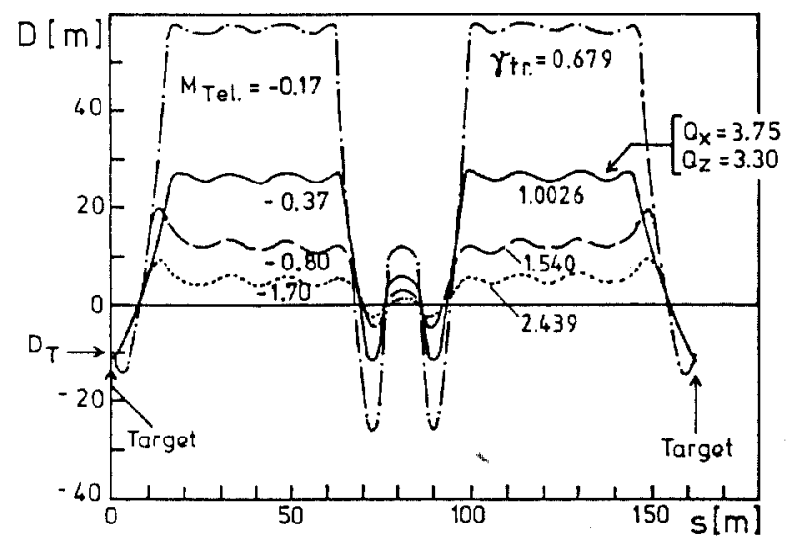

Figure 3: Dispersion D along the ring for four different target telescope settings.

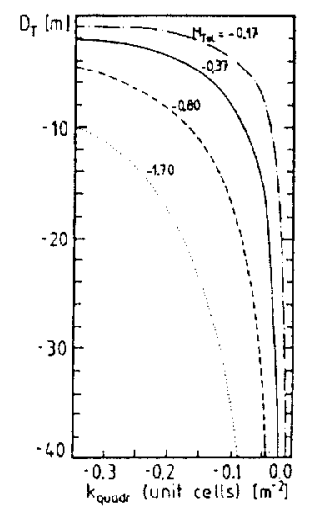

Figure 4:

Target dispersion $D_{T}$ as function of the quadrupole strength in the unit cell for the telescope settings shown in fig. 3.

\section{Injection and Extraction}

The injection into the cosy ring will be accomplished at first from the existing cyclotron JULIC ${ }^{4}$ which delivers unpolarized $p, d, \alpha$ and ${ }^{3} \mathrm{He}$-particles from an internal source with energies from 22.5 to 45 $\mathrm{MeV} / \mathrm{N}$. Light iuns up to ${ }^{20} \mathrm{Ne}$ will be avajlable in 1986 from the ISIS system ${ }^{5}$ (external injection and ECR source) which is under construction. Stripping injection of e.g. $\mathrm{H}_{2}^{+}$will allow typically $10^{9}$ particles in the ring. For other particles such as a's stacking by kicker and septum magnets has to be applied.
For high luminosity proton experiments the SNQ-LINAC will be an ideal injector which enables the ring to be filled to the space charge limit ( $>10^{11}$ particles. This LINAC provides a 250 us long bunch of $6 \cdot 10^{9}$ protons every $5 \mathrm{~ns}$ so that 20 bunches in $100 \mathrm{~ns}$ will fill the ring. This is smaller than the revolution time of about 600 ns allowing stripping injection of $\mathrm{H}^{-}$or kicker-septum injection of protons up to the space charge limit within less than a revolution.

With the injection scheme as shown in Fig. 1 counter-clockwise circulation allows measurement of the forward angles $\left(\theta_{1} \leqq 90^{\circ}\right.$ ) with the high resolution spectrometer BIG KARL. Special modifications of BIG KARL are necessary for the most forward angles.

\section{Phase Space Cooling}

Phase space cooling will be an important part of the COSY ring. Without cooling the increase of the brilliance (= beam current/emittance) in the recirculator mode ${ }^{6}$ is 1 imited to a factur of $10-10^{5}$ dependent on energy and particle. In this mode the beam passes many times through an internal target of typically 50 $100 \mu \mathrm{g} / \mathrm{cm}^{2}$. While the average energy loss can be corrected by a small RF acceleration multiple scattering causes an emittance growth until the resolution becomes so poor that the beam has to be dumped in a beam stop. Electron cooling will be able to keep the emittance of the beam small if the target thickness is typically smaller than $0.1 \mu \mathrm{g} / \mathrm{cm}^{2}$. This implies the use of very thin targets e.g. gas jet targets.

The initial electron cooling system will provide an electron beam with energies up to $100 \mathrm{keV}$ and electron densities in the order of $10^{8} \mathrm{~cm}^{-3}$. It is oriented at the LEAR electron cooler ${ }^{7}$ and will allow the cooling of beams between injection energy and nearly $200 \mathrm{MeV}$. The length of the cooling section will be about $2 \%$ of the ring cirumference. An upgrading of the electron cooling system to $300 \mathrm{xV}$ is intended for the second stage.

Stochastic cooling ${ }^{8}$ with amplifier bandwidths of $200-700 \mathrm{MHz}$ is an order of magnitude slower than electron cooling for the same number of particles. With the possible development ${ }^{9}$ of $2-8 \mathrm{GHz}$ bandwidths the cooling time can be decreased by a factor of 10 . Because for large emittances stochastic cooling works faster than electron cooling a combination of both cooling methods will be installed in the COSY ring.

\section{Acceleration}

The basic requirements for acceleration in cosy are given in table II.

\section{Table II Acceleration parameters for protons}

injection examples

\begin{tabular}{lcll}
$E[\mathrm{MeV}]$ & 40 & 200 & 1500 \\
$\mathrm{~B} \rho[\mathrm{T} \mathrm{m}]$ & 0.924 & 2.150 & 7.507 \\
accel.time[sec] & - & 0.19 & 1.0 \\
$\beta$ & 0.283 & 0.566 & 0.923 \\
revolution time[us] & 1.940 & 0.970 & 0.595 \\
\hline field ramp. [T/sec] & \multicolumn{2}{c}{0.94} \\
$\Delta$ E per turn [KeV] & & \multicolumn{2}{c}{1.1} \\
\hline
\end{tabular}


Ferrite loaded tuned cavities with a peak voltage of $V-4 \mathrm{kV}$ and a frequency change of a factor 3.3 perform the acceleration.

\section{Construction Steps and Modes of Operation}

Construction and operation of the storage ring should be realized in the following steps:

1) Construction of the storage ring with RF-structures for the correction of the average energy loss in the target, matching and installation of the BIG KARL spectrometer and a recoil spectrometer at the ring, injection from the cyclotron. Operation of the system in the recirculation mode.

2) Installation of ferrite loaded tunable RF-structures. Operation in the extended energy range.

3) Installation and operation of an electron cooler $\left(E_{p} \lesssim 100 \mathrm{keV}\right)$ for $E_{p} \lesssim 180 \mathrm{MeV}$. Use of gas jet and atomic beam targets. Development of new target techniques. Installation of stochastic cooling for higher energies.

4) Injection from the SNQ-LINAC. RF-Cavities for the energy range up to $1.5 \mathrm{GeV}$.

For the future requirements, electron cooling for higher energies can be developed and installed in the ring. Depending on the construction step and the experimental requirements the ring can be operated in different modes:

- as recirculator 10

- as beam cooler

- with energy variation

with externa? beam

as stretcher for proton beams of the SNQ-LINAC.

The properties in the cooler ring for nuclear and atomic physics are summarized in the following:

- Very high luminosity far experimegnts with extremely thin targets ( $\$ 10^{1}$ atoms $/ \mathrm{cm}^{2}$ ).

- Variahle duty factor up to $100 \%$ (DC beam).

- Fine tuning of the primary energy (1 MeV/min) with the electron cooler.

- Very accurate absolute energy definition by the measurement of electron energy.

A stripping injection efficiency of about $50 \%$, i.e. measurement during injection.

- No beam stop in the recirculation mode (internal target) hence reduced background.

\section{Experiments}

In addition to the spectrometer target area with specially designed variable beam properties, there are several places in the ring which will be used as experimental areas $E$ (see fig. 1).

With the beam properties of COSY a variety of nuclear and atomic physics experiments becomes possible. With proton energies up to $1.5 \mathrm{GeV} \operatorname{COSY}$ will be an ideal instrument to study subnuclear degrees of freedom in nuclei.

In the following some of the experimental possibilities are listed:

- Fine energy tuning for sharp resonances and threshold energies.

- Measurement of heavy recoil nuclei and reaction products.

- Tagging of secondary beams by recoil measurements (e.g. tagged neutrons).

- Nuclear reactions with extremely high resolution.

- Coincidence experiments with high luminosity and qood eneray resolution in three body reactions.

- Experiments with polarized atomic beams as target.

- Storage of exotic reaction products (e.g. tritons ${ }^{11}$ ).

- Nuclear reactions and r-spectroscopy with low background and very sinall cross sections.

- Spin excitations in the window of 150-300 MeV.

- Production of neutrons with good energy resolution by $7_{L i}(p, n)$ or ${ }^{12} C(p, n)$ reactions at $0^{0}$ for neutron induced reactions.

- Low lying hole states at $E_{p}=400-500$ MeV.

- Polarization transfer experiments, spin-spin interactions with high resolution.

The authors wish to express their gratitude to K. Kilian (CERN), P. Lefévre (CERN), D. Mohl (CERN), and R.E. Pollock (IUCF, Bloomington) for their valuable contributions to the discussions of this project.

\section{References}

1) Workshop on Electron Cooling, Bad Honnef, May 1982, Juil-Spez-159, Kernforschungsanlage Jïlich, ed. G.P.A. Berg, W. Hürlimann, J.G.M. Römer, J. Debrus, F. Hinterberger, and S.A. Martin.

G. Berg, G. Gaul. H. Hagedoorn, A. Hardt, J.A. van der Heide, F. Hinterberger, Max Huber, R. Jahn, $S$. Martin, T. Mayer-Kuckuk, F. Osterfeld, H. Paetz gen. Schieck, D. Prasuhn, G. Riepe, M. Rogge, P. von Rossen, 0.W.B. Schult, J. Speth, P. Turek, Studie zum Bau eines kombinierten Kühler-Synchrotron-Rings an der KFA Jiilich (COSY-Studie), JiilSpez-242, Febr. 1984, Kernforschungsanlage Jül ich

2) S.A. Martin, A. Hardt, J. MeiBburger, G.P.A. Berg, U. Hacker, W. Hürlimann, J.G.M. Römer, T. Sagefka, A. Retz, O.W.B. Schult, K.L. Brown, and K. Halbach, Nucl. Instr. Meth. 214281 (1983)

3) H.L. Hagedoorn and N.F. Verster, Nucl. Instr. Meth. 18, 201 (1962).

4) L. Aldea, W. Bräutigam, R. Brings, C. MayerBöricke, J. Reich and P. Wucherer, Proc. 9th Int. Conf. on Cyclotrons and their Applications, Caen (France) (1981) p. 103.

5) H.-G. Mathews, H. Beuscher, W. Krauss-Vogt, ECRIon-Source Development at the Jilich Cyclotron, Proc. Int. Ion Engineering Congress, Kyoto 1983.

6) S.A. Martin, D. Prasuhn, W. Schott, and C.A. Wiedner, A Storage Ring for the JULIC Cyclotron, contribution to the IX Al] Union National Conference on Particle Accelerators, Dubna, USSR, Oct. 1984.

7) A. Wolf, Status and Perspectives of the Electron Cooling Device for LEAR under Construction at CERN, LEAR Workshop - Tignes, Jan. 1985

8) D. Möhl, Comparison between Electron Cooling and Stochastic Cooling, contribution to ECOOL84, Karlsruhe, Sept. 1984.

9) E. Jones, Progress on ACOL, III. LEAR Workshop 1985.

10) S.A. Martin, D. Prasuhn, W. Schott, C.A. Wiedner, A Storage Ring for the Jlll IC Cyclotron, to be published in Nucl. Instr. Meth.

11) R. Jahn, Tritonenstrahl in COSY, COSY-Arbeitstreffen, 26.-27. Okt. 1983, ed. G.P.A. Berg, D. Prasunn, J.G.M. Römer, Jül-Spez-253, KFA Jülich 1984. 